\title{
Optimization and Calculation of Pt-Sn Phase Diagram
}

\author{
Hu Jieqiong ${ }^{a}$, Xie Ming $^{b}$, Sun Shaoxia ${ }^{c}$, ZhangJiming ${ }^{d}$, Yang Youcai ${ }^{\mathrm{e}}$, Liu Man \\ men $^{f}$, Wang Song ${ }^{g}$, Wang Saibei ${ }^{\text {h }}$
}

\author{
State Key Laboratory of Advanced Technologies for Comprehensive Utilization of Platinum Metals, \\ Kunming Institute of Precious Metals, Kunming 650106, China \\ ajoanr8210@163.com, bpowder@ipm.com.cn, c'sunshaoxia@ipm.com.cn, ${ }^{d} z j m \_z a n e @ 163 . c o m,{ }^{e} y$ \\ angycai@126.com,

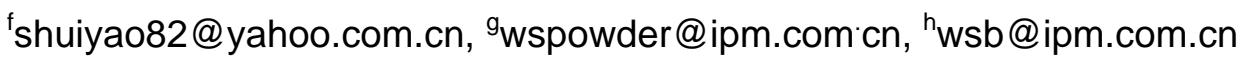

Keywords: Pandat software; Thermodynamic calculations; Thermodynamic parameters; Pt-Sn system

\begin{abstract}
Optimization and calculation of the phase diagram of Pt-Sn binary system were presented by Pandat software.The Gibbs free energy of the pure component ( $\mathrm{Au}$ and $\mathrm{Pt}$ ) was described by the expression of the SGTE (Science Group Thermodata Europe) database, Using the solid solution phase thermodynamic model to describe the liquid and solid solution phases. Using the PanOptimizer optimization platform and the latest experimental data, the parameters for each phase of the Pt-Sn binary system were optimized. Both calculated phase diagram and thermodynamic properties agree well with most of relative experimental data.
\end{abstract}

\section{Introduction}

Precious metal alloys widely used in aviation, spaceflight, navigation, missile, rocket, atomic energy, microelectronics, chemical, petrochemical, glass fiber, exhaust gas purification and in metallurgical industry, due to their unique physical and chemical properties, therefore it is called "the vitamin of modern industry" and "modern new metal"[1-3].

In the precious metal alloys, Pt-Sn binary system has a certain application in brazing[4-5], heterogeneous catalysis[6-7], dental and jewelry industry[8-10]. Pt-Sn binary alloy is one of the important materials of Pt base welding wire [11]. For Pt-Sn system has these special fields of application, so the calculation of phase diagram to describe the multiple systems containing Pt-Sn system and phase equilibrium relations become very valuable. Durussel etc.[12] first determined phase diagram of Pt-Sn binary system, and later Doerinckel[13] Podkopajew[14], Ferro[15] and Schaller[16] successively studied the phase diagram of Pt-Sn binary system. Boer et.al[17] has carried on the semi-quantitative calculation on solid phase and liquid phase of the binary system. Anres etc[11] restudied the determination of the system and the formation enthalpy of the system. Su etc [18] also did the thermodynamic calculation and optimization to phase diagram of Pt-Sn binary system, but by the limiting condition such as the development of experimental conditions and the thermodynamic model, therefore, it is necessary for further optimization and calculation of Pt-Sn binary phase diagram.

\section{The present research situation of Pt-Sn binary system}

Durussel etc[12] studied the intermetallic compounds of Pt-Sn binary system in temperature range of $500-1500{ }^{\circ} \mathrm{C}$ by electron microprobe analysis, differential thermal analysis and X-ray diffraction (XRD) analysis in 1994, and pointed out that the decomposition temperatures of Pt3Sn $\left(1402^{\circ} \mathrm{C}\right)$ and PtSn $\left(1276^{\circ} \mathrm{C}\right)$ and Pt2Sn3 (898 $\left.{ }^{\circ} \mathrm{C}\right)$, PtSn2 $\left(748^{\circ} \mathrm{C}\right)$ and PtSn4 $\left(540^{\circ} \mathrm{C}\right)$ intermetallic compound, and by analysis of metallograph and XRD quenching samples, points out that PtSn and PtSn3 with similar structure(P63/mmc), they also calculate the lattice parameters of all intermetallic compounds of Pt-Sn system. Anres etc[11] measured the liquid formation enthalpy of Pt-Sn system in temperature range $870 \mathrm{~K}$ to $1660 \mathrm{~K}$ and the formation enthalpy of PtSn and Pt3Sn 
through the calorimetric experiment in 1998. The experiments showed that PtSn had two different structure in low temperature and high temperature, at low temperature the formation enthalpy of $\mathrm{PtSn}$ is $-74 \mathrm{~kJ} \mathrm{~mol}^{-1}$, the formation enthalpy of PtSn at high temperature is $63.5 \mathrm{~kJ} \mathrm{~mol}^{-1}$, and the formation enthalpy of Pt3Sn is $-55.3 \mathrm{~kJ} \mathrm{~mol}^{-1}$. Su etc[18] calculated the phase diagram of Pt-Sn system and researched the thermodynamic parameters of Pt-Sn system in 2001. They used self consistent thermodynamic model parameters to describe the phase equilibrium on the system, and the mesophase of system used stoichiometric ratio model, the phase diagram and thermodynamic parameters optimized by the calculation agreed well with the experimental data. The main existing phase in the Pt-Sn binary system were liquid phase, intermediate phase and FCC phase, the equilibrium reaction temperature and component from different references of Pt-Sn system as shown in table 1.

Table 1 A comparison of calculated and experimental equilibria in the Pt-Sn system

\begin{tabular}{|c|c|c|c|}
\hline Reaction & $\begin{array}{l}\text { Temperature, } \\
\mathrm{K}\end{array}$ & \begin{tabular}{l}
\multicolumn{1}{c}{ Liquid } \\
composition \\
(at.\% Sn)
\end{tabular} & Reference \\
\hline \multirow[t]{3}{*}{ Liq. $\rightarrow \operatorname{Pt}(\mathrm{fcc})+\mathrm{Pt}_{3} \mathrm{Sn}$} & 1638 & Uncertain & \\
\hline & $1643 \pm 2$ & 0.196 & \\
\hline & 1656.2 & 0.215 & This study \\
\hline \multirow[t]{3}{*}{ Liq. $\rightarrow \mathrm{Pt}_{3} \mathrm{Sn}$} & 1693 & 0.25 & \\
\hline & 1675 & 0.25 & \\
\hline & 1674.83 & 0.251 & This study \\
\hline \multirow[t]{3}{*}{ Liq. $\rightarrow \mathrm{Pt}_{3} \mathrm{Sn}+\mathrm{PtSn}$} & 1343 & 0.40 & \\
\hline & 1340 & 0.394 & \\
\hline & 1341.16 & 0.382 & This study \\
\hline \multirow[t]{3}{*}{ Liq. $\rightarrow$ PtSn } & 1578 & $0.485-0.50$ & \\
\hline & 1549 & 0.5 & \\
\hline & 1548.61 & 0.498 & This study \\
\hline \multirow{3}{*}{ Liq. $+\mathrm{PtSn} \rightarrow \mathrm{Pt}_{2} \mathrm{Sn}_{3}$} & 1121 & 0.75 & \\
\hline & 1171 & 0.728 & \\
\hline & 1171.01 & 0.726 & This study \\
\hline \multirow[t]{3}{*}{ Liq. $+\mathrm{Pt}_{2} \mathrm{Sn}_{3} \rightarrow \mathrm{PtSn}_{2}$} & 1018 & 0.81 & \\
\hline & 1021 & 0.812 & \\
\hline & 1021.84 & 0.825 & This study \\
\hline \multirow[t]{3}{*}{ Liq. $+\mathrm{PtSn}_{2} \rightarrow \mathrm{PtSn}_{4}$} & 795 & 0.95 & \\
\hline & 813 & 0.95 & \\
\hline & 812.48 & 0.939 & This study \\
\hline \multirow[t]{3}{*}{ Liq. $\rightarrow \mathrm{PtSn}_{4}+\beta(\mathrm{Sn})$} & 501 & 0.995 & \\
\hline & 501 & 0.994 & \\
\hline & 505.01 & 0.999 & This study \\
\hline
\end{tabular}

\section{The selection, optimization and calculation of the thermodynamic model}

\section{The thermodynamic model of each binary system.}

Gibbs free energy of pure components

Gibbs free energy expression of pure components used $G_{i}^{0, \phi}(T)=G_{i}^{\phi}(T)-H_{i}^{S E R}$, which element i ( $\mathrm{i}=\mathrm{Pt}, \mathrm{Sn})$ is componentof $\Phi$ phase $(\Phi=\mathrm{FCC}(\mathrm{Pt}, \mathrm{Sn}), \mathrm{BCT} \mathrm{Sn}$ and liquid), the relationship between free energy and the temperature is:

$$
G_{i}^{0, \phi}(T)=a+b T+c T \ln T+d T^{2}+e T^{3}+f T^{-1}+g T^{7}+h T^{-9}
$$

Type $H_{i}^{S E R}$ is the molar enthalpy of pure element $\mathrm{i}$ at $298.15 \mathrm{~K}$ and under 1 atmospheric pressure, $\mathrm{T}$ is the absolute temperature, $\mathrm{a}, \mathrm{b}, \mathrm{c}, \mathrm{d}, \mathrm{e}, \mathrm{f} . .$. is the fitting undetermined coefficients, Gibbs free 
energy expression of pure component (Pt and Sn) using SGTE (Science Group Thermodata Europe) expression in the database [19].

Thermodynamics model of liquid phase.

In actual solution, atoms binding energy often influenced by temperature, composition and other factors, the interaction are not always the constant, therefore, to modify the interaction parameters, making it the function related to the temperature and composition, in order to achieve the more accurate description of the liquid phase and the FCC phase in Pt-Sn system, choose the subregular solution model to describe the Gibbs free energy, the Gibbs free energy expression is:

$$
G^{L}\left(T,\left\{y_{i}\right\}\right)=\sum_{i} y_{i} G_{i}^{0, L}(T)+R T \sum_{i} y_{i} \ln y_{i}+\sum_{i, j>i} y_{i} y_{j} \sum_{v} L_{i, j}^{L, v}\left(y_{i}-y_{j}\right)^{v}
$$

Type $y_{i}$ is the mole fraction in the liquid phase of element $i, i(j)$ on behalf of each phase, namely Pt, Sn and PtSn. $G_{P t}^{0, L}(T)$ and $G_{S n}^{0, L}(T)$ in formula (2) is the gibbs free energy of Pt, Sn liquid phase, adopts the SGTE database expression [20]. The gibbs free energy $G_{P t S n}^{0, L}(T)$ using the following formula:

$$
G_{P t S n}^{0, L}(T)=G_{P t}^{0, L}(T)+G_{S n}^{0, L}(T)+a_{P t S n}^{L}+b_{P t S n}^{L} T
$$

Type $a_{P t S n}^{L}$ and $b_{P t S n}^{L}$ is formation enthalpy and entropy of PtSn respectively, the two parameters were optimized.

The thermodynamic model of solid phase (Pt)

The computation formula gibbs free energy of face-centered cubic phase $\mathrm{Pt}$ is as follows:

$$
G^{(P t)}=x_{P t} G_{P t}^{0, f c c}+x_{S n} G_{S n}^{0, f c c}+R T\left(x_{P t} \ln x_{P t}+x_{S n} \ln x_{S n}\right)+x_{P t} x_{S n} L_{P t, S n}^{0,(P t)}
$$

Thermodynamics model of intermediate phase

Pt-Sn system have five intermediate phase, namely PtSn, PtSn2, Pt2Sn3, Pt3Sn, PtSn4, among them PtSn crystal structure belongs to the hexagonal system, has the NiAs type structure, the space group is P63/mmc, crystal parameters are $a=b=4.104 \AA, c=5.436 \AA, \alpha=\beta=90 \stackrel{\circ}{\circ}, \gamma=120^{\circ}$, cell contains four atoms; Crystal structure of PtSn2 belongs to the cubic system, the space group is Fm-3m, crystal parameters are $a=b=c=6.4331 \AA, \alpha=\beta=\gamma=90^{\circ}$, cell contains 12 atoms; Pt2Sn3 crystal structure belongs to the hexagonal system, the space group is $P 63 / \mathrm{mmc}$, crystal cell parameters are $a=b=4.325 \AA, c=12.934 \AA, \alpha=\beta=90^{\circ}, \gamma=120^{\circ}$, the cell contains 10 atoms; Pt3Sn crystal structure belong to cubic system, with AuCu3 type structure, space group is Pm-3m, crystal cell parameters are $a=b=c=4 \AA, \alpha=\beta=\gamma=90^{\circ}$, the cell contains 4 atoms; PtSn 4 crystal structure belongs to the orthogonal system, space group is ABA2, crystal cell parameters are $\mathrm{a}=6.397 \AA, \mathrm{b}=$ $6.426 \AA, c=11.381 \AA, \alpha=\beta=\gamma=90^{\circ}$, the cell contains 20 atoms [20-23]. The computation formula of gibbs free energy of five intermediate phase is:

$$
G^{P t_{p} S n_{q}}(T)=\frac{p}{p+q} G_{P t}^{0, f c c}(T)+\frac{q}{p+q} G_{S n}^{0, b c t}(T)+A^{\prime}+B^{\prime} T
$$

Type A 'and B' is optimized parameters.

\section{The optimized and calculated of thermodynamic parameters.}

PanOptimizer is the parameters module in $\mathrm{C} / \mathrm{C}++$ software environment, which evaluated experiment thermodynamics, kinetics and thermal physical model, not only can be used as a thermodynamic model parameters optimization tools, as well as optimization of thermal physical properties and dynamic model parameters [24]. Using the recent related experimental data, and combined with PanOptimizer optimization platform, optimized each phase parameters. Raub.E, S.N.Tripathi, T.B.Massalski, J.Korb, Okamoto.H and S.R.Bharadwaj successively has carried on the experiment and thermodynamics calculation for Pt-Sn binary system. Among them the work of S.R.Bharadwaj and J.Korb is more comprehensive and reliable, so optimization mainly chose their experimental data and SGTE proposed data in 2010.

Optimization carried by PanOptimizer program. First, optimization the phase parameters one by one, and then overall optimization. The Optimized parameters as shown in table 2. 
Table 2 Evaluated thermodynamic parameters for Pt-Sn system

\begin{tabular}{|c|c|c|}
\hline Phase type & Phase & Thermodynamic parameters $\left(\mathrm{Jmol}^{-1}\right)$ \\
\hline \multirow{5}{*}{$\begin{array}{l}\text { Solid solution } \\
\text { phase }\end{array}$} & \multirow{4}{*}{ Liquid } & $G_{P t S n}^{0, L i q}=-94663.3+G_{P t}^{0, L i q}+G_{S n}^{0, \text { Liq }}$ \\
\hline & & $L_{P t, P t S n}^{0, L i q}=-71062.3+25.6461 T$ \\
\hline & & $L_{P t, P t S n}^{1, L i q}=-10754.4$ \\
\hline & & $L_{P t S n, S n}^{0, L i q}=-33610.0+18.8021 T$ \\
\hline & $(\mathrm{Pt})$ & $L_{P t, S n}^{0,(P t)}=-125000-2 T$ \\
\hline \multirow{5}{*}{$\begin{array}{l}\text { Intermediate } \\
\text { phase }\end{array}$} & $\mathrm{Pt}_{3} \mathrm{Sn}$ & $G^{P t_{3} S n}=-35826.2-0.5 T+\left(\frac{3}{4}\right) G_{P t}^{0, f c c}+\left(\frac{1}{4}\right) G_{S n}^{0, b c t}$ \\
\hline & PtSn & $G^{P t S n}=-59500+4.3833 T+\left(\frac{1}{2}\right) G_{P t}^{0, f c c}+\left(\frac{1}{2}\right) G_{S n}^{0, b c t}$ \\
\hline & $\mathrm{Pt}_{2} \mathrm{Sn}_{3}$ & $G^{P t_{2} S n_{3}}=-52240+4.83 T+\left(\frac{2}{5}\right) G_{P t}^{0, f c c}+\left(\frac{3}{5}\right) G_{S n}^{0, b c t}$ \\
\hline & $\mathrm{PtSn}_{2}$ & $G^{P t S n_{2}}=-45160+4.02 T+\left(\frac{1}{3}\right) G_{P t}^{0, f c c}+\left(\frac{2}{3}\right) G_{S n}^{0, b c t}$ \\
\hline & $\mathrm{PtSn}_{4}$ & $G^{P t S n_{4}}=-29000+2.4 T+\left(\frac{1}{5}\right) G_{P t}^{0, f c c}+\left(\frac{4}{5}\right) G_{S n}^{0, b c t}$ \\
\hline
\end{tabular}

\section{Discussion and analysis}

The calculated phase diagram is shown in figure 1. For comparison, the phase diagram calculation and optimization by Su etc is also shown in figure 2. By contrast figure 1 and figure 2, Pt-Sn binary phase diagram calculated by Su etc[18] with Pandat software[25] in the Sn-rich area more than $900{ }^{\circ} \mathrm{C}$ has miscibility phenomenon in liquid phase. But this area of miscibility is actually not exist, nor related experiment verified, so this study abandoned the liquid miscibility, and the liquid area was redefined, introduced liquid phase PtSn, the gibbs free energy of liquid Pt, Sn, calculated by SGTE database expression and gibbs free energy of PtSn liquid can adopt the above formula (3) to calculate. The functions used in the calculation are shown in table 3.

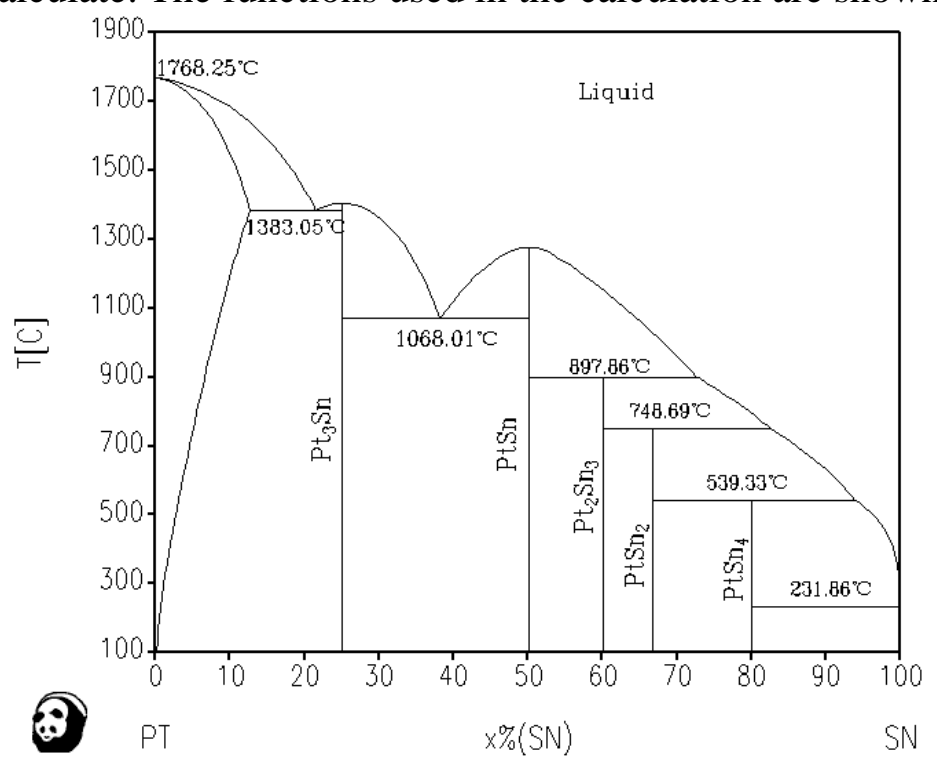

Fig.1. Calculated the Pt-Sn phase by Pandat software 


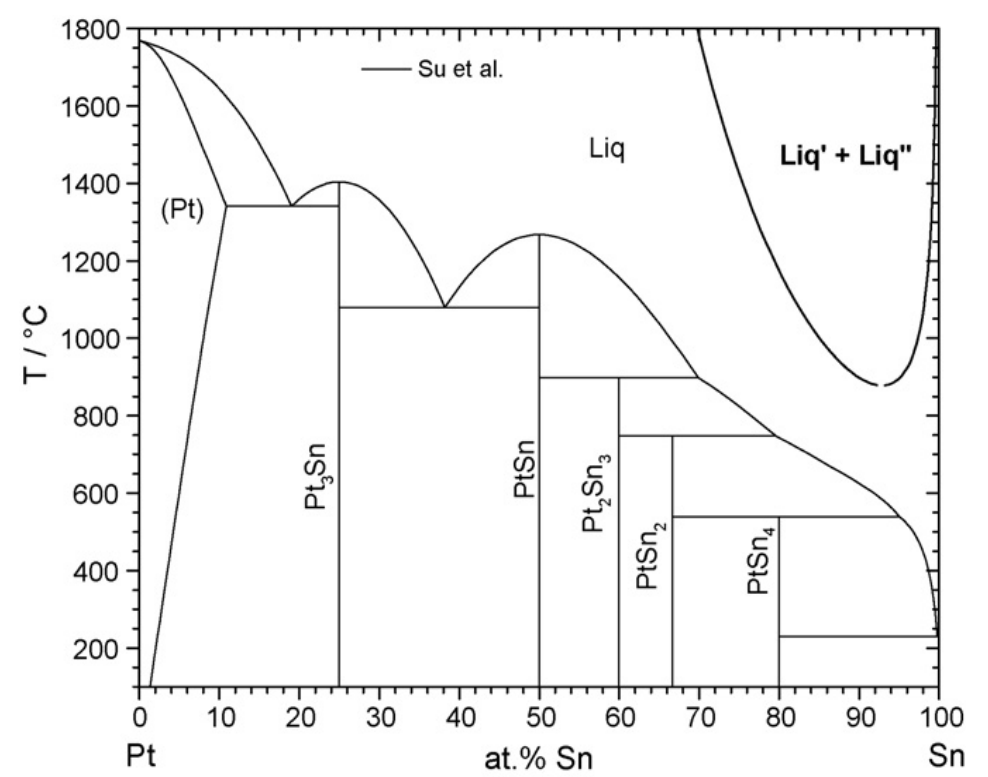

Fig.2. The phase diagram of Pt-Sn system calculated by $\mathrm{Su}^{[18]}$

Table 3 Functions used in the calculation procedure

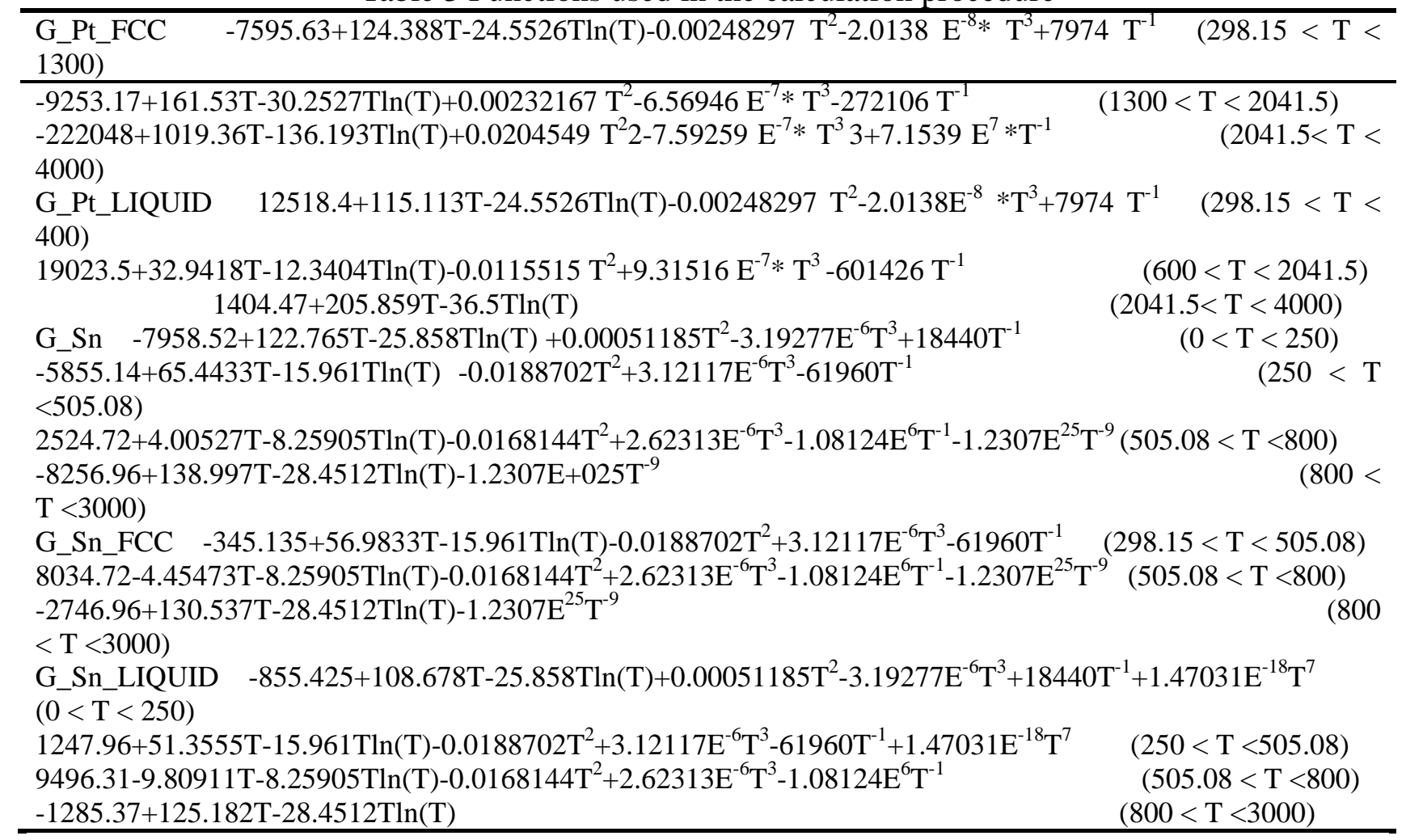




\section{Conclusion}

Using the latest experimental data and the thermodynamic model optimized the parameters of Pt-Sn system, In Pt-Sn system, there is no liquid immiscible area, introduced PtSn into liquid when optimize the system. According to the optimization parameters calculated the Pt-Sn binary phase diagram, the calculated phase diagram are good agreement with the most recent phase diagram in terms of zero variable reaction temperature and ingredients, at the same time, the thermodynamic properties was also coincident.

\section{Acknowledgement}

In this paper, the research was sponsored by the National Nature Science Foundation of China (U1302272, 51267007, 51461023, 51164015); the fund of the Collaborative Innovation Center of Rare and Precious Metals Advanced Materials(2014XT02); Key Project of Yunnan on Applied Fundamental Research (2011FA026); Innovative Team of Yunnan Province (2012HC027); the Innovative Team of Kunming city (2012-01-01-A-R-07-0005)

\section{References}

[1] $\mathrm{Xu}$ Chao, $\mathrm{Xu}$ Yongqing, Yang Yongjun, etal. The application of Au/Sn eutectic bonding technology in MEMS packaging[J]. Micronanoelectronic Technology,2014, 51(2):131-135.

[2] Ma Juan, Sun Hanjun, Zhao Hong, etal. Electrocatalytic Performance of Carbon Supported Pt-Sn Catalyst Prepared with Direct Thermo-decomposition Method for Ethanol Oxidation [J]. Chemical Journal of Chinese Universities,2011, 32(12):2856-2860.

[3] Cai Jiangnan, Yang Fanger, Qiu Kaifeng,etal. Novel electrical contact materials Ag/La1-xSrxCoO3[J]. Electronic Components and Materials,2014, 33(7):1-3.

[4]Osamu Wada, Tsugio Kumai.Preferential reaction and stability of the Au - Sn/Pt system: Metallization structure for flip - chip integration[J].Appl. Phys. Lett., 1991, 58(9):908-910

[5] J.F. Kuhmann, C.-H. Chiang, P. Harde, F. Reier, W. Oesterle, I. Urban, A.Klein.Pt thin-film metallization for FC-bonding using SnPb60/40 solder bump metallurgy[J].Materials Science and Engineering: A, 1998, 242(1-2):22-25

[6]R. Burch.Platinum-tin reforming catalysts: I. The oxidation state of tin and the interaction between platinum and tin[J].Journal of Catalysis, 1981, 71(2):348-359

[7]R. Burch.Platinum-tin reforming catalysts: II. Activity and selectivity in hydrocarbon reactions[J].Journal of Catalysis, 1981, 71(2):360-372

[8] B.Kempf, P. Spencer, J. Hauelt. The application of platinum and the interaction between platinum and tin[J].Zeitschrift fu?r Metallkunde, 1995, 86(9):603-607

[9] B.Kempf, S. Schmauder.The stability of the Sn-Pt system and its applications [J].Gold Bull., 1998, 31(2):51-57

[10] T.Biggs, S.S. Taylor, E. van der Lingen.Pt-Sn system and its applications [J].Platinum Met., 2005, 49(1):2-15 [11] P.Anres, M. Gaune-Escard, J.P. Bros, E. Hayer.Enthalpy of formation of the (Pt-Sn) system[J].Journal of Alloys and Compounds, 1998, 280(1-2):158-167

[12] Ph.Durussel, R. Massara, P. Feschotte. Le système binaire Pt-Sn[J].Journal of Alloys and Compounds, 1994, 215(1-2):175-179

[13] F.Doerinckel.Metallographische Mitteilungen aus dem Institut für anorganische Chemie der Universit?t G?ttingen über einige Platinlegierungen[J].Zeitschrift für anorganische und allgemeine Chemie, 1907, 54(1):349-357 
[14] N.Podkopajew, Zh. Russ.Phase diagram of Pt-Sn system [J].Fiz. Khim. Obshch., 1908, 40(1):249-256

[15] R.Ferro, R. Capelli, A. Borsese, S. Delfino. in: Rendiconti della Classe di Scienze fisiche, matematiche e naturali[M].matematiche e naturali,, 1973, LIV(serie VIII.):4-20

[16] H.J. Schaller.Thermal properties of Pt-Sn system[J].Zeitschrift für Physikalische Chemie, 1978, 112(2):85-91

[17] F.R. de Boer, R. Boom, W.C.M. Mattens, A.R. Miedema, A.K.Niessen.Cohesions in Metals[J].Cohesions in Metals, 1988, 1(1):20-40

[18] X.Su, F. Yin, M. Huang, et al.. Thermodynamic assessment of the Pt-Sn system[J].Journal of Alloys and Compounds, 2001, 325(1):109-112

[19] A.T. Dinsdale.SGTE data for pure elements[J].Calphad, 1991, 15(4):317-425

[20]Wei Zhou, Lijuan Liu, Baoling $\mathrm{Li}$, et al.Structural,elastic and electronic properties of intermetallics in the Pt-Sn system: A density functional investigation[J].Computational Materials Science, 2009, 46(4):921-931

[21] Shelton K L, Merewether P A, Skinner B J.Phases and phase relations in the system Pd-Pt-Sn[J].Canadian Mineralogist, 1981, 19(1):599-605

[22] Charlton J S, Cordey H M, Harris I R.A study of the 119Sn Moessbauer isomer shifts in some platinum-tin and gold-tin alloys[J].Journal of the Less-Common Metals, 1970, 20(2):105-112

[23] Schubert K, Pfisterer H.Die Kristallstruktur von Pt2Sn3[J].Zeitschrift fuer Metallkunde, 1949, 40(3):105-112

[24]S.L.Chen.Pandat8.0 User’s Guide[M] .United States : CompuTherm LLC， 2008.

[25] S-L. Chen, S. Daniel, F. Zhang, Y.A. Chang, W.A. Oates, R. Schmid-Fetzer.[J].J. Phase Equilib. Diff., 2001, 22(2):373-378 